Research Paper

\title{
Clinical Impact of Vitamin K Dosing on Sorafenib Treatment for Hepatocellular Carcinoma
}

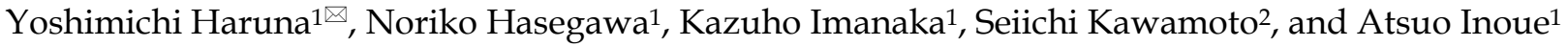 \\ 1. Department of Gastroenterology and Hepatology, Osaka General Medical Center, Osaka, Japan \\ 2. Department of Diagnostic Imaging, Osaka General Medical Center, Osaka, Japan \\ $\triangle$ Corresponding author: Yoshimichi Haruna, Department of Gastroenterology and Hepatology, Osaka General Medical Center, 3-1-56 Bandaihigashi, \\ Sumiyoshiku, Osaka 558-8558, Japan. Fax No.: (81) 6-6606-7000; E mail address: apple-123-grape@ab.auone-net.jp \\ (C) Ivyspring International Publisher. This is an open access article distributed under the terms of the Creative Commons Attribution (CC BY-NC) license \\ (https://creativecommons.org/licenses/by-nc/4.0/). See http://ivyspring.com/terms for full terms and conditions.
}

Received: 2016.12.24; Accepted: 2017.04.01; Published: 2017.07.05

\begin{abstract}
Background: Some researchers have suggested that vitamin $\mathrm{K}$ enhances the antitumor effect of sorafenib for hepatocellular carcinoma (HCC) in vitro and in vivo. In this study, we examined the clinical impact of vitamin $\mathrm{K}$ dosing for sorafenib treatment.

Methods: Twenty-nine out of 65 patients treated with sorafenib for HCC were simultaneously dosed with vitamin $\mathrm{K}$. We retrospectively investigated progression-free survival (PFS) and overall survival (OS) in the vitamin K-dosed group and sorafenib alone group. We also examined the changes in serum des- $\gamma$-carboxy prothrombin (DCP) levels, which vitamin $\mathrm{K}$ is involved with.

Results: The median PFS was prolonged in the sorafenib + vitamin $K$ group compared with the sorafenib alone group (6.0 months and 2.0 months, respectively; $P<0.001$, hazard ratio $[H R]: 0.25$ ). The median OS was also significantly extended ( 12.5 months vs. 10.0 months; $P=0.009$, HR: 0.47 ). Despite suppressed tumor growth, serum DCP levels had increased in cases of disease-controlled patients in the sorafenib alone group 8 weeks after the beginning of treatment, $(2.28 \pm 0.91$ to $2.64 \pm 1.03, P=0.048)$. In contrast, the serum $\mathrm{DCP}$ levels of the sorafenib + vitamin $\mathrm{K}$ group had declined both in patients with controlled disease and in patients with progressive disease ( $1.97 \pm 0.57$ to $1.29 \pm 0.28, \mathrm{P}=0.002$ and $2.90 \pm 1.32$ to $1.78 \pm 0.53, \mathrm{P}=0.034$, respectively).
\end{abstract}

Conclusions: To the best of our knowledge, this is the first clinical report showing enhanced antitumor action of sorafenib by vitamin K. Our clinical findings suggest that vitamin K may have the synergistic effect by suppressing production of DCP, a tumor growth and angiogenesis factor.

Key words: hepatocellular carcinoma, vitamin K, sorafenib, des- $\gamma$-carboxy prothrombin, tumor ischemia

\section{Introduction}

Hepatocellular carcinoma (HCC) is globally the third leading cause of cancer-related mortality $[1,2]$. Early-stage HCC is suitable for surgical resection or locoregional treatment (radiofrequency ablation or percutaneous ethanol infusion), and a favorable prognosis can be obtained (5-yr survival: 40-70\%). Multinodular HCC (intermediate stage) is indicated for transarterial chemoembolization (TACE). However, advanced-stage HCC with extrahepatic spread (nodal metastasis or other organ metastasis), vascular invasion, or TACE-failure is treatable only with sorafenib systemic chemotherapy (Nexavar,
Bayer HealthCare Pharmaceuticals, Leverkusen, Germany) [3].

Sorafenib, a multikinase inhibitor, is a molecular-targeting anti-cancer agent that inhibits the serine-threonine kinase Raf and the tyrosine kinase activity of vascular endothelial growth factor (VEGF) receptors, leading to suppression of cancer cell proliferation and tumor angiogenesis $[4,5]$. However, the anti-cancer effect of sorafenib is limited despite its frequent adverse events. Llovet et al. reported that median overall survival (OS) was 10.7 months in the sorafenib group and 7.9 months in the placebo group 
in a clinical study of HCC patients in Europe, America and Australia [6]. Cheng et al. reported that median OS was 6.5 months in sorafenib-treated patients and 4.2 months in control patients in a clinical trial in the Asia-Pacific region [7]. These studies showed that the median OS in sorafenib-treated HCC patients was prolonged by a few months. Thus, more efficient anticancer agents or modified sorafenib therapies are desired.

Some reports have shown that vitamin $\mathrm{K}$ can inhibit cell proliferation and the growth of HCC in vitro and in vivo [8-11]. Furthermore, Habu et al. reported that vitamin $\mathrm{K}$ dosing suppressed hepatocarcinogenesis among cirrhotic patients [12]. Some authors have suggested that vitamin $\mathrm{K}$ dosing could prevent HCC recurrence after curative treatment [13,14]. In contrast, Yoshida, et al. threw doubt upon the preventive effect of vitamin $\mathrm{K}$ on HCC recurrence [15].

On the other hand, Wei et al. reported that a combination of vitamin $\mathrm{K}$ and sorafenib strongly induced growth inhibition and apoptosis of HCC cells due to inhibition of the Raf/mitogen-activated protein kinase kinase/Phospho-extracellular signal regulated kinase. They showed marked tumor growth suppression of transplanted HCC in rats treated with vitamin $\mathrm{K}$ plus sorafenib compared with rats treated with sorafenib alone or vitamin $\mathrm{K}$ alone [16]. Carr et al. suggested that the c-Met-PI3K-Akt signaling pathway mediates inhibitory c-Raf phosphorylation that may induce the synergistic action of vitamin K and sorafenib [17]. A report from another group also showed that sorafenib plus vitamin $\mathrm{K}$ treatment markedly inhibited migration and proliferation of HCC cells in vitro [18].

Vitamin $\mathrm{K}$ supplementation is believed to sustain bone mineral density and to prevent osteoporotic fracture $[19,20]$. Therefore, in Japan, vitamin $\mathrm{K}$ is often administered to cirrhotic patients as a precaution against osteoporosis. We have observed some cases of simultaneous sorafenib and vitamin $\mathrm{K}$ dosing. In this study, we retrospectively examined the clinical impact of vitamin $\mathrm{K}$ dosing on sorafenib treatment for hepatocellular carcinoma.

Vitamin $\mathrm{K}$ is also involved with des- $\gamma$-carboxy prothrombin (DCP) production. We also examined how vitamin $\mathrm{K}$ dosing influenced the serum DCP levels during treatment.

\section{Patients and Methods}

\section{Patients}

We investigated all 65 patients who underwent sorafenib treatment with HCC stages of Barcelona Clinic Liver Cancer (BCLC)-B or BCLC-C in the Osaka General Medical Center from May 29, 2009 until May
31, 2016 [21,22]. The patients had had no indication for surgical operation, transarterial embolization (TACE) or radio-frequency ablation. Forty-one patients $(63 \%)$ with advanced stage (BCLC-C) were treated with sorafenib according to the EASL-EORTC clinical practice guidelines: Management of hepatocellular carcinoma [22]. Additional 24 patients (37\%) with intermediate stage (BCLB-B) who had been given a TACE failure status were also treated with sorafenib. TACE failure status was defined as more than two consecutive incomplete necrosis results (more than $50 \%$ of the tumor stain remaining) observed within the treated tumors by response-evaluation computed tomography (CT) 4 weeks after TACE or more than two consecutive appearances of a new lesion (recurrence) observed in the liver by response-evaluation CT 4 weeks after TACE [24]. Based on our sorafenib dosing criteria, an initial dose of sorafenib was given at $800 \mathrm{mg}$ daily, whereas 400 $\mathrm{mg}$ was administered to patients who were older than 80 years, weighed less than $50 \mathrm{~kg}$ or had Child-Pugh status B to avoid adverse events due to overdosing. Forty-three patients $(66 \%)$ were given an initial dose of $800 \mathrm{mg}$ of sorafenib, while 22 patients (34\%) received an initial dose of $400 \mathrm{mg}$ of sorafenib. Forty-eight patients (74\%) had Eastern Cooperative Oncology Group (ECOG) performance status (PS) 0, $11(17 \%)$ had PS 1, and $6(9 \%)$ had PS 2 before sorafenib administration. Regarding Child-Pugh status at baseline, 54 (83\%) had Child-Pugh A, and 11 (17\%) had Child-Pugh B. Twenty-one (32\%) showed macroscopic vascular invasion, and 25 (38\%) had extrahepatic spread (lymph node: 9, bone: 6, lung: 6, adrenal gland: 4, spleen: 1, peritoneum: 1). Twenty-nine $(45 \%)$ out of the 65 patients had simultaneous dosing of sorafenib and vitamin $\mathrm{K}$ (Gla-kay, Eizai Co., Ltd., Tokyo, Japan). Vitamin K dosing began in 5 patients prior to the start of sorafenib treatment, began concurrently in 21 patients, and began after sorafenib treatment in 3 patients.

\section{Outcomes and assessments}

In all cases, radiologic tumor evaluation was performed according to the modified Response Evaluation Criteria in Solid Tumors (RECIST) [25] by dynamic contrast-enhanced CT or EOB magnetic resonance imaging, within 2 weeks before the start of sorafenib dosing, and was repeated every 4 to 8 weeks after the start of treatment until tumor progression. The radiologic review was independently performed by a radiologist and hepatologists. Progression-free survival (PFS) was defined as the time from the start of sorafenib dosing to radiologic progression. OS was assessed from the date of sorafenib dosing until the 
date of death from any cause. The disease control rate was shown as the percentage of patients with complete response, partial response or stable disease that was maintained for at least 4 weeks. Safety was assessed using Common Terminology Criteria for adverse events version 3.0.

The serum DCP levels were tested by ECLIA (Wako Pure Chemical Industries, Ltd., Osaka, Japan). We examined the changes in serum DCP levels before and 8 weeks after the beginning of treatment.

All patients provided written informed consent for the study before the start of sorafenib dosing. The study was approved by the ethics committee of Osaka General Medical Center and complied with the Declaration of Helsinki and local laws.

\section{Statistical analysis}

The Kaplan-Meier method and the log-rank test were used to analyze the impact of vitamin $\mathrm{K}$ dosing on PFS and OS of sorafenib-treated patients. The Cox proportional-hazards model was used to assess the interaction between PFS or OS and characteristics at baseline. The Chi-square test, Fisher's exact test, Student's $t$ test and Mann-Whitney U test were used for analysis of baseline characteristics, disease control rates and adverse events. To evaluate the change in serum DCP levels, the paired t-test was performed after logarithmic transformation.

\section{Results}

\section{Impacts of vitamin $\mathrm{K}$ dosing and baseline characteristics on the PFS and OS of sorafenib-treated patients}

We evaluated the impacts of vitamin $\mathrm{K}$ dosing and baseline characteristics, including Child-Pugh status, ECOG performance status, presence of extrahepatic spread of the HCC, presence of macroscopic vascular invasion, age and initial dose of sorafenib, on the PFS and OS of the sorafenib-treated patients. Multivariate analysis with the use of Cox proportional-hazard model showed that vitamin $\mathrm{K}$ dosing $(\mathrm{P}<0.001$, hazard ratio $[\mathrm{HR}]: 0.16,95 \% \mathrm{CI}$ 0.08-0.32) and presence of macroscopic vascular invasion ( $\mathrm{P}=0.004, \mathrm{HR}: 2.55, \quad 95 \%$ CI 1.34-4.82) influenced PFS of the sorafenib-treated patients significantly (Table 1A). It also showed that the vitamin $\mathrm{K}$ dosing $(\mathrm{P}=0.003$, HR: $0.38,95 \% \mathrm{CI}$ $0.20-0.73)$, ECOG performance status $(\mathrm{P}=0.005$, HR: $0.36,95 \%$ CI $0.17-0.73$ ) and presence of macroscopic vascular invasion $(\mathrm{P}<0.001, \mathrm{HR}: 3.81,95 \% \mathrm{CI}$ 2.00-7.26) influenced OS significantly (Table 1B). It was revealed that the vitamin $\mathrm{K}$ dosing during sorafenib treatment was one of independent factors to improve the prognosis of the patients.
Table 1. Multivariate analysis for the outcome of sorafenib treatment with the use of Cox proportional-hazard model

\begin{tabular}{lll}
\hline & Hazard Ratio (95\% CI) & P Value \\
\hline $\begin{array}{lll}\text { A: Progression-free Survival } \\
\text { Vitamin K dosing }\end{array}$ & $0.16(0.08-0.32)$ & $<0.001$ \\
Child-Pugh status (A vs. B) & $0.95(0.46-1.95)$ & 0.88 \\
ECOG performance status (0 vs. 1 & $0.86(0.45-1.61)$ & 0.64 \\
or 2) & & \\
Extrahepatic spread & $0.96(0.54-1.71)$ & 0.89 \\
Macroscopic vascular invasion & $2.55(1.34-4.82)$ & 0.004 \\
Age & $0.97(0.94-1.00)$ & 0.06 \\
Initial dose of sorafenib & $0.65(0.35-1.21)$ & 0.18 \\
& & \\
B: Overall Survival & & 0.003 \\
Vitamin K dosing & $0.38(0.20-0.73)$ & 0.53 \\
Child-Pugh status (A vs. B) & $0.77(0.34-1.75)$ & 0.005 \\
ECOG performance status (0 vs. 1 & $0.36(0.17-0.73)$ & \\
or 2) & & 0.28 \\
Extrahepatic spread & $1.42(0.75-2.71)$ & $<0.001$ \\
Macroscopic vascular invasion & $3.81(2.00-7.26)$ & 0.60 \\
Age & $1.01(0.98-1.05)$ & 0.98 \\
Initial dose of sorafenib & $1.01(0.54-1.89)$ & \\
\hline
\end{tabular}

ECOG: Eastern Cooperative Oncology Group.

Table 2. Baseline Characteristics of the Patients

\begin{tabular}{|c|c|c|c|}
\hline Variables & $\begin{array}{l}\text { Sorafenib+vitamin } \\
K \\
(n=29)\end{array}$ & $\begin{array}{l}\text { Sorafenib } \\
\text { alone } \\
(n=36)\end{array}$ & $\begin{array}{l}P \\
\text { value }\end{array}$ \\
\hline Age, mean \pm S.D. & $72.6 \pm 8.4$ & $74.4 \pm 8.1$ & 0.38 \\
\hline \multicolumn{4}{|l|}{ Gender, $\mathbf{n}(\%)$} \\
\hline Male & $22(76)$ & $26(72)$ & \multirow[t]{2}{*}{0.74} \\
\hline Female & $7(24)$ & $10(28)$ & \\
\hline \multicolumn{4}{|l|}{ ECOG performance status, $n(\%)$} \\
\hline 0 & $22(76)$ & $26(72)$ & \multirow{3}{*}{0.84} \\
\hline 1 & $5(17)$ & $6(17)$ & \\
\hline 2 & $2(7)$ & $4(11)$ & \\
\hline \multicolumn{4}{|l|}{ Cause of disease, $\mathbf{n}(\%)$} \\
\hline HCV infection & $20(69)$ & $23(64)$ & \multirow{4}{*}{0.97} \\
\hline HBV infection & $4(14)$ & $5(14)$ & \\
\hline Alcohol & $3(10)$ & $5(14)$ & \\
\hline Other & $2(7)$ & $3(8)$ & \\
\hline \multicolumn{4}{|l|}{ BCLC stage, n (\%) } \\
\hline B (intermediate) & $10(34)$ & $14(39)$ & \multirow[t]{2}{*}{0.71} \\
\hline C (advanced) & $19(66)$ & $22(61)$ & \\
\hline Macroscopic vascular invasion, $\mathrm{n}(\%)$ & $10(34)$ & $11(31)$ & 0.53 \\
\hline Extrahepatic spread, n (\%) & $10(34)$ & $15(42)$ & 0.55 \\
\hline \multicolumn{4}{|l|}{ Child-Pugh status, n (\%) } \\
\hline A & $25(86)$ & $29(81)$ & \multirow[t]{2}{*}{0.40} \\
\hline $\mathrm{B}$ & $4(14)$ & $7(19)$ & \\
\hline \multicolumn{4}{|l|}{ Alpha-fetoprotein, ng/mL } \\
\hline Median & 97.4 & 95.4 & \multirow{2}{*}{0.93} \\
\hline Range & 1.9-105146.7 & $1.6-133133.9$ & \\
\hline \multicolumn{4}{|l|}{ Initial dose of sorafenib } \\
\hline 800mg daily, n (\%) & $19(66)$ & $24(67)$ & 0.92 \\
\hline
\end{tabular}

HBV: hepatitis B virus. HCV: hepatitis C virus. BCLC: Barcelona Clinic Liver Cancer. ECOG: Eastern Cooperative Oncology Group.

\section{Baseline characteristics of the sorafenib + vitamin $K$ and the sorafenib alone groups}

There were no relevant differences between the sorafenib + vitamin $\mathrm{K}$ group and the sorafenib alone group in baseline characteristics, including age, gender, ECOG performance status, cause of disease, BCLC stage, macroscopic vascular invasion, extrahepatic spread, Child-Pugh status, serum alpha fetoprotein levels or initial dose of sorafenib (Table 2). 


\section{Response rates and disease control rates}

No complete response was observed in the study. In the sorafenib + vitamin K group, 10 (34\%) patients obtained a partial response (PR), 10 (34\%) had stable disease (SD) and 9 (31\%) had progressive disease (PD). In contrast, in the sorafenib alone group, $5(14 \%)$ had a PR, $5(14 \%)$ had SD and $25(69 \%)$ had PD. Disease control rates were $69 \%$ in the sorafenib + vitamin $\mathrm{K}$ group and were $23 \%$ in the sorafenib alone group $(\mathrm{P}<0.001)$ (Table 3$)$.

Table 3. Response rates by modified RECIST

\begin{tabular}{lll}
\hline & $\begin{array}{l}\text { Sorafenib+vitamin K } \\
(\mathbf{n = 2 9 )}\end{array}$ & $\begin{array}{l}\text { Sorafenib alone } \\
\mathbf{( n = 3 6 )}\end{array}$ \\
\hline Complete response $\mathbf{n}(\mathbf{0})$ & $0(0)$ & $0(0)$ \\
Partial response $\mathbf{n}(\%)$ & $10(34)$ & $5(14)$ \\
Stable disease $\mathbf{n}(\%)$ & $10(34)$ & $5(14)$ \\
Progressive disease $\mathbf{n}(\%)$ & $9(31)$ & $25(69)$ \\
Not assessable $\mathbf{n}(\mathbf{0})$ & $0(0)$ & $1(3)$ \\
aDisease control rate $\mathbf{n}(\mathbf{0})$ & $20(69.0)$ & $8(22.9)$ \\
\hline a P<0.001 & \\
RECIST: Response Evaluation Criteria in Solid Tumors
\end{tabular}

\section{Progression-free survival and overall survival}

The median PFS was prolonged in the sorafenib + vitamin K group compared with the sorafenib alone group (6.0 months, 95\% CI 3.7-8.3 vs. 2.0 months, $95 \%$ CI 1.4-2.6; $\mathrm{P}<0.001$ by log-rank test), and the hazard ratio was 0.25 (95\% CI 0.14-0.46) (Fig. 1A).

The median OS was also significantly extended in the sorafenib + vitamin K group compared with the sorafenib alone group (12.5 months, 95\% CI 5.4-19.6 vs. 10.0 months, $95 \%$ CI 5.9-14.1; $\mathrm{P}=0.009)$, and the hazard ratio was 0.47 (95\% CI 0.26-0.85) (Fig. 1B).

\section{Serum DCP change}

We examined the changes in serum DCP levels before and 8 weeks after the beginning of treatment. In the sorafenib + vitamin K group, both patients with PR or SD and patients with PD had significant decline of the serum DCP levels (mean \pm S.D. [Log $\mathrm{mAU} / \mathrm{mL}]: 1.97 \pm 0.57$ to $1.29 \pm 0.28, \mathrm{P}=0.002$ and 2.90 \pm 1.32 to $1.78 \pm 0.53, \mathrm{P}=0.034$, respectively) (Fig. $2 \mathrm{~A}$, Fig. 2B). In contrast, in the sorafenib alone group, the serum DCP levels increased significantly in patients with PR or SD as well as in patients with PD (mean \pm S.D. [Log $\mathrm{mAU} / \mathrm{mL}]: 2.28 \pm 0.91$ to $2.64 \pm 1.03, \mathrm{P}=$ 0.048 and $2.65 \pm 0.95$ to $3.14 \pm 1.17, \mathrm{P}=0.020$, respectively) (Fig. 2C, Fig. 2D).

(B)

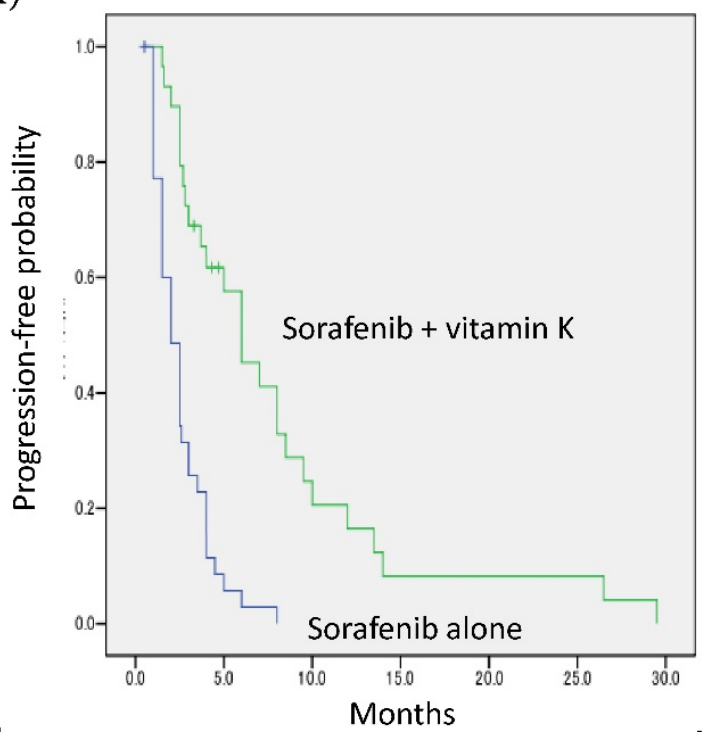

No. at risk

No. at risk

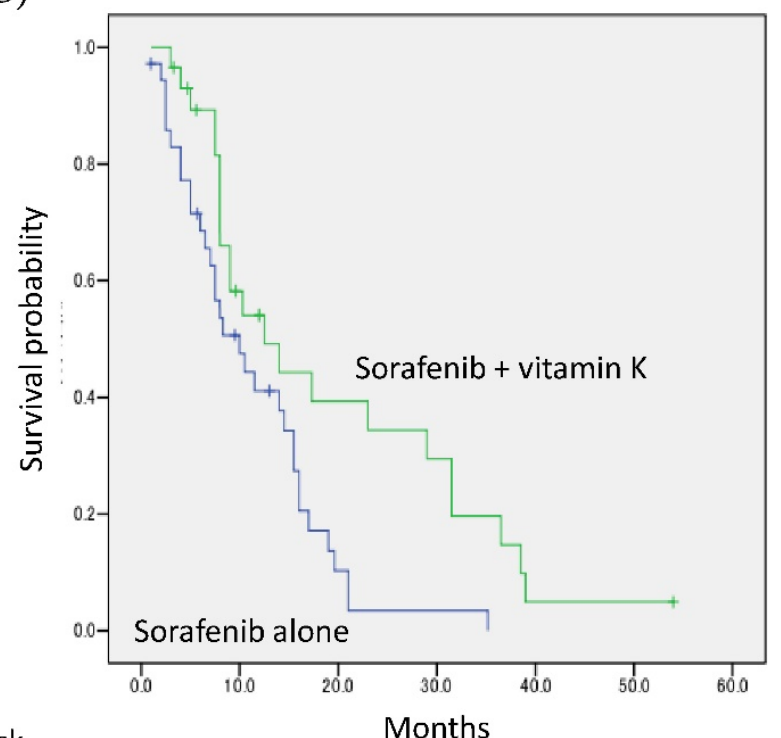

$\begin{array}{lrrrrrrrrrrrrr}\text { Sorafenib + vitamin K } & 29 & 24 & 14 & 9 & 8 & 7 & 6 & 4 & 1 & 1 & 1 & 0 & 0 \\ \text { Sorafenib alone } & 36 & 25 & 15 & 10 & 3 & 1 & 1 & 1 & 0 & 0 & 0 & 0 & 0\end{array}$

Figure 1. Kaplan-Meier curves of progression-free survival (A) and overall survival (B). (A) The median progression-free survival time was prolonged in the vitamin K-dosed group $(n=29)$ compared with the sorafenib alone group $(n=36)(6.0$ months, $95 \% \mathrm{Cl} 3.7-8.3$ months vs. 2.0 months, $95 \% \mathrm{Cl} 1.4-2.6$ months; $\mathrm{P}<0.001$ by log-rank test), and the hazard ratio was $0.25(95 \% \mathrm{Cl} 0.14-0.46)$. (B) The median overall survival time was also significantly extended (12.5 months, $95 \% \mathrm{Cl} 5.4-19.6$ months vs. 10.0 months, $95 \% \mathrm{Cl} 5.9-14.1$ months; $\mathrm{P}=0.009)$, and the hazard ratio was $0.47(95 \% \mathrm{Cl} 0.26-0.85)$. 
(A)

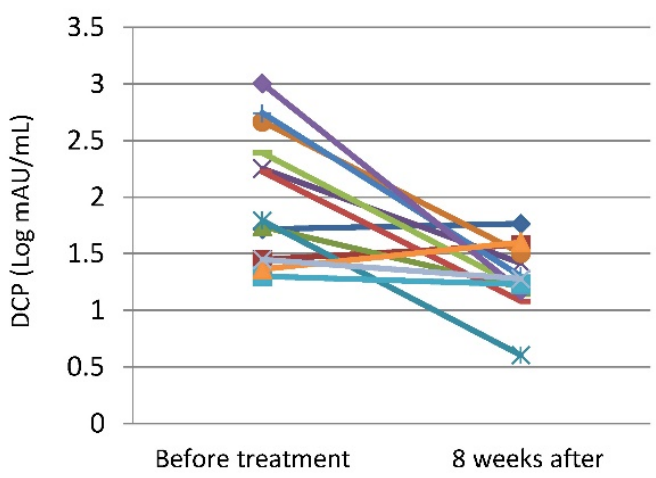

(C)

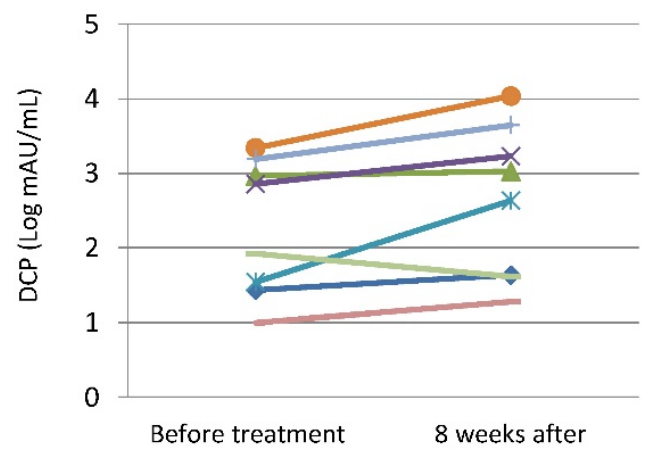

(B)

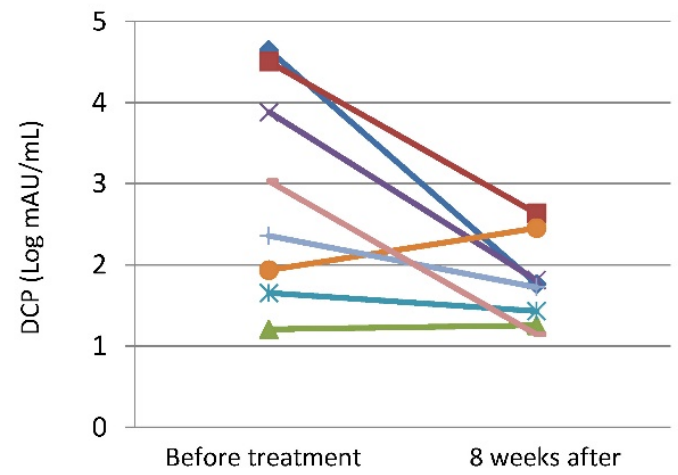

(D)

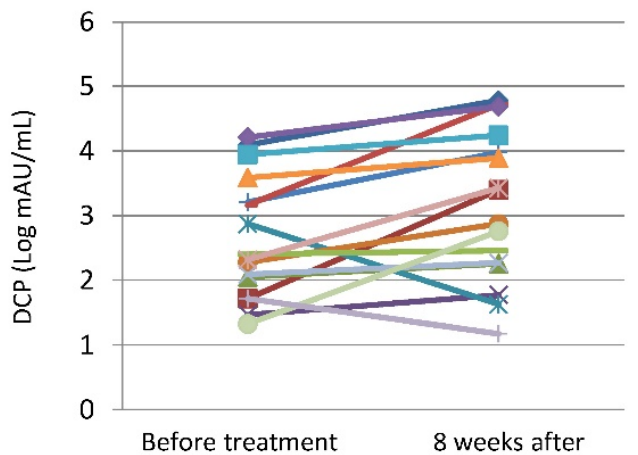

Figure 2. Change in serum DCP levels before and 8 weeks after the beginning of treatment. (A) Patients with PR or SD of the sorafenib + vitamin $K$ treatment group $(n=14)$. (B) Patients with PD of the sorafenib + vitamin K group $(n=8)$. (C) Patients with PR or SD of the sorafenib alone treatment group ( $n=8)$. (D) Patients with PD of the sorafenib alone treatment group $(n=16)$. In the sorafenib + vitamin K group, 22 patients were available because they concurrently started sorafenib and vitamin $\mathrm{K}$ dosing, and serum DCP levels were tested just before and 8 weeks after the beginning of the treatment. In the sorafenib alone group, 24 patients were available because their serum DCP levels were tested just before and 8 weeks after the beginning of the treatment. The change of DCP levels (mean $\pm S . D$. [Log $\mathrm{mAU} / \mathrm{mL}])$ were $1.97 \pm 0.57$ to $1.29 \pm 0.28(P=0.002)(A), 2.90 \pm 1.32$ to $1.78 \pm 0.53(P=0.034)(B), 2.28 \pm 0.91$ to $2.64 \pm 1.03(P=0.048)(C)$, and $2.65 \pm 0.95$ to $3.14 \pm 1.17(P=0.020)(D)$. $T$ o evaluate the change in serum DCP levels, a paired t-test was performed for log-transformed measures with normal distribution.

Table 4. Incidence (percent) of drug-related adverse events ${ }^{\mathrm{a}}$

\begin{tabular}{|c|c|c|c|c|c|c|}
\hline Adverse event & $\begin{array}{l}\text { Sorafe } \\
\text { K }\end{array}$ & nib+vitamir & Sorafe & enib alon & P value & \\
\hline & $\begin{array}{l}\text { Any } \\
\text { Grade }\end{array}$ & Grade 3/4 & $\begin{array}{l}\text { Any } \\
\text { Grade }\end{array}$ & $\begin{array}{l}\text { Grade } \\
3 / 4\end{array}$ & $\begin{array}{l}\text { Any } \\
\text { Grade }\end{array}$ & $\begin{array}{l}\text { Grade } \\
3 / 4\end{array}$ \\
\hline Overall incidence & 83 & 45 & 89 & 69 & 0.36 & 0.045 \\
\hline $\begin{array}{l}\text { Hand-foot } \\
\text { skin reaction }\end{array}$ & 45 & 7 & 47 & 25 & 0.85 & 0.052 \\
\hline Liver dysfunction & 24 & 7 & 33 & 25 & 0.42 & 0.052 \\
\hline Diarrhea & 24 & 3 & 14 & 6 & 0.29 & 0.58 \\
\hline $\begin{array}{l}\text { Pancreatic amylase } \\
\text { elevation }\end{array}$ & 24 & 14 & 19 & 11 & 0.65 & 0.50 \\
\hline Hypertension & 14 & 0 & 28 & 6 & 0.17 & 0.30 \\
\hline Rash/desquamation & 38 & 7 & 19 & 11 & 0.10 & 0.45 \\
\hline Fatigue & 7 & 0 & 8 & 3 & 0.60 & 0.55 \\
\hline Hoarseness & 10 & 0 & 8 & 0 & 0.56 & NA \\
\hline Alopecia & 3 & 0 & 8 & 0 & 0.39 & NA \\
\hline
\end{tabular}

a Drug-related adverse events that occurred in at least $5 \%$ of patients in either group were listed. They were assessed using Common Terminology Criteria for adverse events version 3.0

NA: not applicable

\section{Safety}

Table 4 shows all drug-related adverse events that occurred in at least $5 \%$ of patients in either group. Hand-foot skin reaction, liver dysfunction, diarrhea, serum pancreatic amylase elevation, hypertension, rash/desquamation, fatigue, hoarseness, and alopecia were observed during medication. Overall, the incidence of drug-related adverse events of any grade was $83 \%$ in the sorafenib + vitamin K group and $89 \%$ in the sorafenib alone group. The incidence of each adverse event was similar in the two groups. However, the incidence of grade $3 / 4$ hand-foot skin reaction and grade $3 / 4$ liver dysfunction in the sorafenib + vitamin $\mathrm{K}$ group tended to be lower than in the sorafenib alone group. Overall, the incidence of grade 3/4 adverse events was significantly lower in the sorafenib + vitamin $\mathrm{K}$ group $(45 \%$ vs. $69 \%$, $\mathrm{P}=0.045)$.

\section{Discussion}

Some studies showed that vitamin $\mathrm{K}$ dosing enhanced the antitumor effect of sorafenib in vitro and in vivo in animal studies [16-18]. However, there has been no report about the clinical impact of vitamin $\mathrm{K}$ dosing for sorafenib-treated patients. Some of the patients in this study, whom we treated with sorafenib for $\mathrm{HCC}$, received vitamin $\mathrm{K}$ dosing simultaneously. We retrospectively studied the 
influence of vitamin $\mathrm{K}$ dosing on antitumor action, cancer prognosis and the incidence of adverse events to clarify the impact of vitamin $\mathrm{K}$ dosing during sorafenib treatment. Multivariate analysis with the use of Cox proportional-hazard model revealed that the vitamin $\mathrm{K}$ dosing during sorafenib treatment was one of independent factors to improve the prognosis of the patients, as absence of vascular invasion or preferable ECOG performance status. We found significantly prolonged PFS (2.0 months vs. 6.0 months) and OS (10.0 months vs. 12.5 months) with no relevant difference in baseline characteristics between the sorafenib alone group and the sorafenib + vitamin K group. Regarding safety, we did not observe any difference in incidence of drug-related adverse events between sorafenib-alone group and sorafenib + vitamin K group. The overall incidence of grade $3 / 4$ adverse events was lower in sorafenib + vitamin $\mathrm{K}$ group rather than in sorafenib-alone group.

We have some reports suggesting that DCP, which is one of the popular tumor markers for HCC, is an autologous growth factor for HCC $[26,27]$ and a paracrine factor for tumor angiogenesis [28-30]. DCP released from tumor cells is shown to bind to Met on the tumor cells and kinase insert domain receptor on vascular endothelial cells, resulting in tumor cell proliferation and endothelial cell proliferation and migration. Additionally, $\mathrm{Ma}$ et al. showed that vitamin $\mathrm{K}$ inhibits the growth of hepatocellular carcinoma via a decrease of DCP in vitro [9]. We speculated that the benefit of vitamin $\mathrm{K}$ dosing for sorafenib treatment might be associated with DCP levels from tumor cells. We focused on the change of serum DCP levels during anticancer treatment. The serum DCP levels were increased by sorafenib-alone treatment in disease-controlled patients as well as in patients with PD. The paradoxical phenomenon, DCP elevation despite suppressed tumor growth, could be explained as the following. Vitamin $\mathrm{K}$ is an essential co-factor in prothrombin production from its precursor, DCP. Because HCC tumor cells possess an impaired vitamin $\mathrm{K}$ uptake system, prothrombin production is suppressed due to the lack of vitamin $\mathrm{K}$ inside tumor cells, followed by accumulation and release of DCP [31]. Thus, DCP can be a tumor marker for HCC. Sorafenib deteriorates tumor angiogenesis and puts tumor cells in an ischemic state [4,5]. Murata et al. showed that hypoxia further decreases the vitamin $\mathrm{K}$ uptake of tumor cells and leads to more accumulation and release of DCP [32]. However, augmented DCP release could provide an advantage for tumor cells to survive because DCP works as a tumor growth factor [26,27] and an angiogenesis enhancing factor [28-30]. Sorafenib's antitumor action may be limited by elevation of DCP.
In contrast, we found a remarkable decline in the serum DCP levels both in patients with disease-controlled patients and in patients with PD of the sorafenib + vitamin K group. According to in vitro study by Murata et al., high-dose vitamin K administration elevates the vitamin $\mathrm{K}$ concentration inside tumor cells in a dose-dependent manner and suppresses DCP accumulation and release [31]. Vitamin $\mathrm{K}$ dosing could enhance the antitumor action of sorafenib by suppressing DCP levels.

To the best of our knowledge, this is the first clinical report showing the beneficial impact of vitamin $\mathrm{K}$ dosing during sorafenib treatment for patients with HCC. The vitamin $\mathrm{K}$, a nontoxic agent, might be involved with pathobiology of sorafenib treatment for HCC via DCP production.

\section{Abbreviations}

HCC: hepatocellular carcinoma; OS: overall survival; PFS: progression-free survival; DCP: des- $\gamma$-carboxy prothrombin; BCLC: Barcelona Clinic Liver Cancer; ECOG: Eastern Cooperative Oncology Group; PS: performance status; PR: partial response; SD: stable disease; PD: progressive disease.

\section{Author Contributions}

Conception and design: Yoshimichi Haruna, Atsuo Inoue

Collection and assembly of data: Yoshimichi Haruna, Noriko Hasegawa, Atsuo Inoue

Analysis and interpretation of data: Yoshimichi Haruna, Kazuho Imanaka, Atsuo Inoue, Seiichi Kawamoto

Drafting of the manuscript: Yoshimichi Haruna, Atsuo Inoue

Approval of the final version of the manuscript:

All authors

\section{Competing Interests}

The authors have declared that no competing interests exist.

\section{References}

1. El-Serag HB, Rudolph KL. Hepatocellular carcinoma: epidemiology and molecular carcinogenesis. Gastroenterology. 2007;132:2557-2576.

2. Parkin DM. Global cancer statistics in the year 2000. Lancet Oncol. 2001;2:533-543.

3. Bruix J, Sherman M. Management of hepatocellular carcinoma: An update. Hepatology. 2011;53:1020-1022.

4. Wilhelm SM, Carter C, Tang L, et al. BAY 43-9006 exhibits broad spectrum oral anticancer activity and targets the RAF/MEK/ERK pathway and receptor tyrosine kinases involved in tumor progression and angiogenesis. Cancer Res. 2004;64:7099-7109.

5. Chang YS, Adnane J, Trail PA, et al. Sorafenib (BAY 43-9006) inhibits tumor growth and vasculization and induces tumor apoptosis and hypoxia in RCC xenograft models. Cancer Chemother Pharmacol. 2007;59:561-574.

6. Llovet JM, Ricci S, Mazzaferro V, et al. for the SHARP Investigators Study Group. Sorafenib in advanced hepatocellular carcinoma. N Engl J Med. 2008;359:378-390.

7. Cheng AL, Kang YK, Chen Z, et al. Efficacy and safety of sorafenib in patients in the Asia-Pacific region with advanced hepatocellular carcinoma: a phase III 
randomised, double-blind, placebo-controlled trial. Lancet Oncol. 2009;10:25-34.

8. Otsuka M, Kato N, Shao RX, et al. Vitamin K2 inhibits the growth and invasiveness of hepatocellular carcinoma cells via protein kinase A activation. Hepatology. 2004;40:243-251.

9. Ma M, Qu XJ, Mu GY, et al. Vitamin K2 inhibits the growth of hepatocellular carcinoma via decrease of des-gamma-carboxy prothrombin. Chemotherapy. 2009;55:28-35

10. Azuma K, Urano T, Ouchi $Y$, Inoue S. Vitamin K2 suppresses proliferation and motility of hepatocellular carcinoma cells by activating steroid and xenobiotic receptor. Endcr J. 2009;56:843-849.

11. Li L, Qi Z, Qian J, et al. Induction of apoptosis in hepatocellular carcinoma Smmc-7721 cells by vitamin $\mathrm{K}(2)$ is associated with p53 and independent of the intrinsic apoptotic pathway. Mol Cell Biochem. 2010;342:125-131.

12. Habu D, Shiomi S, Tamori A, et al. Role of vitamin K2 in the development of hepatocellular carcinoma in women with viral cirrhosis of the liver. JAMA. 2004;292:358-361.

13. Mizuta T, Ozaki I, Eguchi Y, et al. The effect of menatetrenone, a vitamin K2 analog, on disease recurrence and survival in patients with hepatocellular carcinoma after curative treatment: a pilot study. Cancer. 2006;106:867-872.

14. Kakizaki S, Sohara N, Sato K, et al. Preventive effects of vitamin K on recurrent disease in patients with hepatocellular carcinoma arising from hepatitis $C$ viral infection. J Gastroenterol Hepatol. 2007;22: 518-522.

15. Yoshida $\mathrm{H}$, Shiratori $Y$, Kudo $M$, et al. Effect of vitamin $\mathrm{K} 2$ on the recurrence of hepatocellular carcinoma. Hepatology. 2011;54:532-540.

16. Wei $G$, Wang $M$, Hyslop $T$, et al. Vitamin $K$ enhancement of sorafenib-mediated HCC cell growth inhibition in vitro and in vivo. Int J Cancer. 2010;127:2949-2958.

17. Carr BI, Wang Z, Wang M, et al. D'Alessandro R, Refolo MG. c-Met-Akt pathway-mediated enhancement of inhibitory c-Raf phosphorylation is involved in vitamin K1 and sorafenib synergy on HCC growth inhibition. Cancer Biol Ther. 2011;12:531-538.

18. Ha TY, Hwang S, Hong HN, et al. Synergistic effect of sorafenib and vitamin K on suppression of hepatocellular carcinoma cell migration and metastasis. Anticancer Res. 2015;35:1985-1995.

19. Knapen MH, Schurgers LJ, Vermeer C. Vitamin K2 supplementation improves hip bone geometry and bone strength indices in postmenopausal women. Osteoporos Int. 2007;18:963-972.

20. Shiraki M, Shiraki Y, Aoki C, et al. Vitamin K2 (menatetrenone) effectively prevents fractures and sustains lumbar bone mineral density in osteoporosis. J Bone Miner Res. 2000;15:515-521.

21. Llovert JM, Burroughs A, Bruix J. Hepatocellular carcinoma. Lancet. 2003;362:1907-1917.

22. Bruix J, Sherman M. Management of hepatocellular carcinoma. Hepatology. 2005;42:1208-1236.

23. European Association for the Study of the Liver European Organization for Research and Treatment of Cancer. EASL-EORTC Clinical Practice Guidelines: Management of hepatocellular carcinoma. J Hepatol. 2012;56:908-943.

24. Kudo M, Izumi N, Kokudo N, et al. for the HCC Expert Panel of Japan Society of Hepatology. Management of hepatocellular carcinoma in Japan: Consensus-based clinical practice guidelines proposed by the Japan Society of Hepatology (JSH) 2010 updated version. Dig Dis. 2011;29:339-364.

25. Lencioni R, Llovet JM. Modified RECIST (mRECIST) assessment for hepatocellular carcinoma. Semin Liver Dis. 2010;30:52-60.

26. Suzuki M, Shiraha $H$, Fujikawa $T$, et al. Des- $\gamma$-carboxy prothrombin is a potential autologous growth factor for hepatocellular carcinoma. J Biol Chem. 2005;280:6409-6415.

27. Zhang YS, Chu JH, Cui SX, et al. Des-gamma-carboxy prothrombin (DCP) as a potential autologous growth factor for the development of hepatocellular carcinoma. Cell Physiol Biochem. 2014;34:903-915.

28. Fujikawa $\mathrm{T}$, Shiraha $\mathrm{H}$, Ueda $\mathrm{N}$, et al. Des-gamma-carboxyl prothrombin-promoted vascular endothelial cell proliferation and migration. J Biol Chem. 2007;282:8741-8748.

29. Gao FJ, Cui SX, Chen MH, et al. Des-gamma-carboxy prothrombin increases the expression of angiogenic factors in human hepatocellular carcinoma cells. Life Sci. 2008;83:815-820.

30. Wang SB, Cheng YN, Cui SX, et al. Des-gamma-carboxy prothrombin stimulates human vascular endothelial cell growth and migration. Clin Exp Metastasis. 2009;26:469-477.

31. Murata K, Sakamoto A. Impairment of clathrin-mediated endocytosis via cytoskeletal change by epithelial to fibroblastoid conversion in HepG2 cells: a possible mechanism of des-gamma-carboxy prothrombin production in hepatocellular carcinoma. Int J Oncol. 2008;33:1149-1155.

32. Murata K, Suzuki H, Okano H, et al. Hypoxia-induced des-gamma-carboxy prothrombin production in hepatocellular carcinoma. Int J Oncol. 2010;36:161-170 